PROCEEDINGS OF THE

AMERICAN MATHEMATICAL SOCIETY

Volume 136, Number 12, December 2008, Pages 4125-4133

S 0002-9939(08)09632-9

Article electronically published on July 23, 2008

\title{
THE RESTRICTED ISOMORPHISM PROBLEM FOR METACYCLIC RESTRICTED LIE ALGEBRAS
}

\author{
HAMID USEFI
}

(Communicated by Gail R. Letzter)

\begin{abstract}
Let $L$ be a restricted Lie algebra with the restricted enveloping algebra $u(L)$ over a perfect field of positive characteristic $p$. The restricted isomorphism problem asks what invariants of $L$ are determined by $u(L)$. This problem is the analogue of the modular isomorphism problem for finite $p$ groups. Bagiński and Sandling have given a positive answer to the modular isomorphism problem for metacyclic $p$-groups. In this paper, we provide a positive answer to the restricted isomorphism problem in case $L$ is metacyclic and $p$-nilpotent.
\end{abstract}

\section{INTRODUCTION}

Let $L$ be a Lie algebra over a field $\mathbb{F}$ of positive characteristic $p$ and denote by ad $: L \rightarrow L$ the adjoint representation of $L$ given by $(\operatorname{ad} x)(y)=[y, x]$, where $x, y \in$ $L$. Recall that $L$ is called restricted if $L$ additionally affords a $p$-map ${ }^{[p]}: L \rightarrow L$, satisfying

(1) $(\operatorname{ad} x)^{p}=\operatorname{ad}\left(x^{[p]}\right)$, for every $x \in L$;

(2) $(\alpha x)^{[p]}=\alpha^{p} x^{[p]}$, for every $x \in L$ and $\alpha \in \mathbb{F}$; and

(3) $(x+y)^{[p]}=x^{[p]}+y^{[p]}+\sum_{i=1}^{p-1} s_{i}(x, y)$, for all $x, y \in L$, where $i s_{i}(x, y)$ is the coefficient of $\lambda^{i-1}$ in $\operatorname{ad}(\lambda x+y)^{p-1}(x)$.

Let $L$ be a restricted Lie algebra and denote by $u(L)$ the restricted enveloping algebra of $L$. The restricted isomorphism problem asks what invariants of $L$ are determined by $u(L)$; i.e. given another restricted Lie algebra $H$ with the property that $u(L) \cong u(H)$, as algebras, can we deduce that $L$ and $H$ have the same invariants? Of course, the strongest invariant of $L$ is its isomorphism type. We have considered the restricted isomorphism problem for abelian restricted Lie algebras in [10. One main result of [10] states that if $L$ is an abelian restricted Lie algebra in the class $\mathcal{F}_{p}$, then the isomorphism type of $L$ is determined. Recall that $L$ is said to be in the class $\mathcal{F}_{p}$ if $L$ is finite-dimensional and there exists an integer $k$ such that $L^{[p]^{k}}=0$. It is also proved in [10] that if $\mathbb{F}$ is algebraically closed, then every finite-dimensional abelian restricted Lie algebra over $\mathbb{F}$ is determined by its enveloping algebra.

Received by the editors September 28, 2007.

2000 Mathematics Subject Classification. Primary 17B35, 17B50; Secondary 20C05.

Key words and phrases. Restricted Lie algebras, metacylic, enveloping algebras, isomorphism problem, modular group algebras. 
The restricted isomorphism problem is the analogue of the modular isomorphism problem for group algebras of finite $p$-groups [8]. This sort of isomorphism problem also makes sense for ordinary Lie algebras and has been considered by David Riley and the present author in [6].

Bagiński 2] and Sandling [7] proved that every metacyclic $p$-group $G$ is determined by its modular group algebra $\mathbb{F}_{p} G$, where $\mathbb{F}_{p}$ denotes the field of $p$ elements. Motivated by this result of Bagiński and Sandling, in this paper we consider the isomorphism problem for metacyclic restricted Lie algebras in the class $\mathcal{F}_{p}$. Recall that a restricted Lie algebra $L$ is called metacyclic if $L$ has a cyclic restricted ideal $I$ such that $L / I$ is cyclic. Our main result is as follows:

Theorem. Let $L \in \mathcal{F}_{p}$ be a metacyclic restricted Lie algebra over a perfect field of positive characteristic. Then $L$ is determined by its enveloping algebra $u(L)$.

Before closing this section, I would like to thank Luzius Grunenfelder for many useful discussions.

\section{Preliminaries}

Let $L$ be a restricted Lie algebra with the restricted enveloping algebra $u(L)$ over a field $\mathbb{F}$ of characteristic $p$. Let $X$ be an ordered basis of $L$ over $\mathbb{F}$. The analogue of the Poincaré-Birkhoff-Witt (PBW) Theorem for restricted Lie algebras (see [3]) allows us to view $L$ as a restricted Lie subalgebra of $u(L)$ in such a way that $u(L)$ has a basis consisting of PBW monomials, that is, monomials of the form

$$
x_{1}^{a_{1}} \ldots x_{t}^{a_{t}}
$$

where $x_{1}<\cdots<x_{t}$ in $X, 0 \leq a_{i} \leq p-1$, and $t$ is a non-negative integer. Henceforth, we identify the [p]-map of $L$ by the exponentiation by $p$ in $u(L)$.

The augmentation ideal, $\omega(L)$, of $u(L)$ is the associative ideal of $u(L)$ generated by $L$. Let $\gamma_{1}(L):=L$. We denote by $\gamma_{n}(L):=\left[\gamma_{n-1}(L), L\right]$ the $n^{\text {th }}$ term of the lower central series of $L$. Hence, $L^{\prime}=\gamma_{2}(L)$. Recall that $L$ is said to be nilpotent if $\gamma_{n}(L)=0$ for some $n$; the nilpotence class of $L$, denoted by $\operatorname{cl}(L)$, is the minimal integer $c$ such that $\gamma_{c+1}(L)=0$. We shall denote by $L_{p}^{\prime}$ the restricted Lie subalgebra of $L$ generated by $L^{\prime}$. For a subset $X$ of $L$ we denote by $\langle X\rangle$ and $\langle X\rangle_{p}$ the Lie subalgebra and the restricted Lie subalgebra generated by $X$, respectively. We consider left-normed commutators, that is,

$$
\left[x_{1}, \ldots, x_{n}\right]=\left[\left[x_{1}, x_{2}\right], x_{3}, \ldots, x_{n}\right] .
$$

An element $x \in L$ is called $p$-nilpotent if there exists some non-negative integer $t$ such that $x^{p^{t}}=0$; the exponent of $x$, denoted by $\exp (x)$, is the least integer $s$ such that $x^{p^{s}}=0$. A $p$-polynomial in $x$ has the form $c_{0} x+c_{1} x^{p}+\cdots+c_{t} x^{p^{t}}$, where each $c_{i} \in \mathbb{F}$. Also, recall that $L$ is $p$-nilpotent if there exists a positive integer $k$ such that $L^{p^{k}}=0$; the exponent of $L$, denoted by $\exp (L)$, is the minimal integer $s$ such that $L^{p^{s}}=0$. We say $L \in \mathcal{F}_{p}$ if $L$ is finite-dimensional and $p$-nilpotent. Note that if $L \in \mathcal{F}_{p}$, then $L$ is nilpotent by Engel's Theorem. It is known that $L \in \mathcal{F}_{p}$ if and only if $\omega(L)$ is nilpotent as an associative ideal of $u(L)$; see [5].

The $n^{\text {th }}$ dimension subalgebra of $L$ is

$$
D_{n}(L)=L \cap \omega^{n}(L)=\sum_{i p^{j} \geq n} \gamma_{i}(L)^{p^{j}},
$$


where $\gamma_{i}(L)^{p^{j}}$ is the restricted subalgebra of $L$ generated by all $x^{p^{j}}, x \in \gamma_{i}(L)$; see [4]. It follows from the defining axioms of $L$ that $(x+y)^{p}=x^{p}+y^{p}$ modulo $\gamma_{p}(\langle x, y\rangle)$. It is not hard to see that $\left[D_{n}(L), D_{m}(L)\right]=\gamma_{m+n}(L) \subseteq D_{m+n}(L)$ and $D_{n}(L)^{p} \subseteq D_{n p}(L)$, for every $m, n \geq 1$.

Throughout this paper, we assume that $L$ and $H$ are restricted Lie algebras and $\varphi: u(L) \rightarrow u(H)$ is an isomorphism. It is proved in [10] that $\varphi$ can be replaced by another isomorphism that preserves the augmentation ideals. So, without loss of generality, we assume that $\varphi(\omega(L))=\omega(H)$. We need the following results from [10.

Lemma 2.1. If $u(L) \cong u(H)$, then the following statements hold.

(1) If $L \in \mathcal{F}_{p}$, then $|\operatorname{cl}(L)-\operatorname{cl}(H)| \leq 1$.

(2) $D_{i}(L) / D_{i+1}(L) \cong D_{i}(H) / D_{i+1}(H)$, for every $i \geq 1$.

We remark that whether or not the nilpotence class of $G$ is determined by $\mathbb{F}_{p} G$ has been considered in recent years. However no major result is reported up-to-date; see 1 .

We note that $u(L)$ has the invariant dimension property; that is, the rank of every $u(L)$-module is uniquely defined. Now let $I$ be a restricted ideal of $L$ and denote by $I^{n}$ the vector space spanned by all $z_{1} z_{2} \ldots z_{n}$, where $z_{i} \in I$. We know that the kernel of the natural map $u(L) \rightarrow u(L / I)$ is equal to $I u(L)=u(L) I$. It follows that $\omega^{n}(I) u(L)=I^{n} u(L)=(I u(L))^{n}$, for every $n \geq 1$. Furthermore, we observe that each quotient $I^{n} u(L) / I^{n+1} u(L)$ has a natural right $u(L / I) \cong u(L) / I u(L)$-module structure given by

$$
\left(u+I^{n+1} u(L)\right) \cdot(z+I u(L))=u z+I^{n+1} u(L),
$$

for every $u \in I^{n} u(L)$ and $z \in u(L)$. The proofs of the following two lemmas are analogous to the corresponding lemmas in [6].

Lemma 2.2. Let $I$ be an ideal of $L$. Then each factor $I^{n} u(L) / I^{n+1} u(L)$ is a free right $u(L / I)$-module of rank $\operatorname{dim}_{\mathbb{F}} \omega^{n}(I) / \omega^{n+1}(I)$.

Lemma 2.3. Suppose that $\operatorname{dim}_{\mathbb{F}} I / D_{2}(I)$ is finite. Suppose further that $J$ is a restricted ideal of $H$ such that $\varphi(I u(L))=J u(H)$. Then $D_{n}(I) / D_{n+1}(I) \cong$ $D_{n}(J) / D_{n+1}(J)$, for every $n \geq 1$.

Using the identity $[a b, c]=a[b, c]+[a, c] b$, which holds in any associative algebra, we can see that $L_{p}^{\prime} u(L)=[\omega(L), \omega(L)] u(L)$. Thus the ideal $L_{p}^{\prime} u(L)$ is preserved by $\varphi$. So we may apply the previous lemma to the case $I=L_{p}^{\prime}$ and $J=H_{p}^{\prime}$.

Corollary 2.4. Suppose that $L$ and $H$ are finite-dimensional restricted Lie algebras such that $u(L) \cong u(H)$. Then, for every positive integer $n$, we have

$$
D_{n}\left(L_{p}^{\prime}\right) / D_{n+1}\left(L_{p}^{\prime}\right) \cong D_{n}\left(H_{p}^{\prime}\right) / D_{n+1}\left(H_{p}^{\prime}\right) .
$$

Lemma 2.5. Let $L \in \mathcal{F}_{p}$ such that $\operatorname{cl}(L)=2$. Then, $\operatorname{dim}_{\mathbb{F}} L_{p}^{\prime p^{t}}$ is determined, for every $t \geq 0$. In particular, the exponent of $L^{\prime}$ is determined.

Proof. Note that, by Lemma 2.1, $H$ is nilpotent of class at most 3. So, $H^{\prime}$ is abelian. Note that $D_{p^{t-1}+1}\left(H_{p}^{\prime}\right)=\cdots=D_{p^{t}}\left(H_{p}^{\prime}\right)=H^{\prime p^{t}}$, for every $t \geq 1$. So, by Corollary 2.4 we have $L_{p}^{\prime} / L^{\prime p} \cong H_{p}^{\prime} / H^{\prime p}$ and

$$
L^{\prime p^{t}} / L^{\prime p^{t+1}} \cong H^{\prime p^{t}} / H^{\prime p^{t+1}}
$$


for every $t \geq 1$. Now let $s=\exp \left(L^{\prime}\right)$. It follows that

$$
H^{\prime p^{s}}=H^{\prime p^{s+1}} \text {. }
$$

But $H$ is $p$-nilpotent. Hence, $H^{\prime p^{s}}=0$. Thus $\exp \left(H^{\prime}\right) \leq \exp \left(L^{\prime}\right)$. It follows then by symmetry that the exponent of $H^{\prime}$ is $s$. A reverse induction on $t$ shows that $\operatorname{dim}_{\mathbb{F}} L_{p}^{\prime p^{t}}$ is determined, for every $t \geq 0$.

Lemma 2.6. Let $L \in \mathcal{F}_{p}$ such that $L_{p}^{\prime}$ is cyclic. The following statements hold.

(1) $\operatorname{cl}(L) \leq 3$.

(2) We have $L^{\prime p^{t}} u(L)=\left(L_{p}^{\prime} u(L)\right)^{p^{t}}$, for every $t \geq 1$.

Proof. Let $u \in L_{p}^{\prime}$ such that $L_{p}^{\prime}=\langle u\rangle_{p}$. Let $z \in L$. There exist coefficients $\alpha_{0}, \ldots, \alpha_{t} \in \mathbb{F}$ such that $[u, z]=\sum_{k=0}^{t} \alpha_{k} u^{p^{k}}$. So, $\left[z, u^{p^{k}}\right]=0$, for every $k \geq 1$. It follows that

$$
\left[u, z^{p^{k}}\right]=\alpha_{0}^{p^{k}-1}[u, z]
$$

for every $k \geq 1$. But $z$ is $p$-nilpotent. Hence, either $\alpha_{0}=0$ or $[u, z]=0$. Thus $[u, z] \in\left\langle u^{p}\right\rangle_{p}$. Hence, $\gamma_{3}(L) \subseteq L^{\prime p}$. Now let $v \in L$. Note that $\left[v, u^{p^{k}}\right]=0$, for every $k \geq 1$. Since $[u, z] \subseteq\left\langle u^{p}\right\rangle_{p}$, we deduce that $[u, z, v]=0$. It follows that $\operatorname{cl}(L) \leq 3$. To prove the second assertion note that $L^{\prime p^{t}} u(L) \subseteq\left(L_{p}^{\prime} u(L)\right)^{p^{t}}$. So we need to prove that $\left(L_{p}^{\prime} u(L)\right)^{p^{t}} \subseteq L^{\prime p^{t}} u(L)$. Note that $[v z, u]=v[z, u]+[v, u] z \in\left\langle u^{p}\right\rangle_{p} u(L)$, for every $v, z \in L$. It follows by the PBW Theorem that $\left[u(L), L_{p}^{\prime}\right] \subseteq\left\langle u^{p}\right\rangle_{p} u(L)$. Hence, for every $t \geq 1$, we have

$$
\left(L_{p}^{\prime} u(L)\right)^{p^{t}}=\left(\langle u\rangle_{p} u(L)\right)^{p^{t}} \subseteq\left(\langle u\rangle_{p}\right)^{p^{t}} u(L)=L^{\prime p^{t}} u(L),
$$

as required.

\section{Metacyclic Restricted Lie Algebras}

A restricted Lie algebra $L$ is called metacyclic if $L$ has a cyclic restricted ideal $I$ such that $L / I$ is cyclic. In other words, there exist generators $x, y \in L$ and some $p$-polynomials $g$ and $h$ such that

$$
h(x) \in\langle y\rangle_{p}, \quad[y, x]=g(y) .
$$

In this section $L$ is a non-abelian metacyclic restricted Lie algebra in the class $\mathcal{F}_{p}$. Let $g(y)=\beta_{0} y+\beta_{1} y^{p}+\beta_{2} y^{p^{2}}+\cdots$. We claim that $\beta_{0}=0$. Suppose otherwise. Then

$$
y=\beta[y, x]+\beta_{1}^{\prime} y^{p}+\beta_{2}^{\prime} y^{p^{2}}+\cdots,
$$

where $\beta \neq 0$. Note that $\left[x, y^{p^{t}}\right]=0$, for every $t \geq 1$. Since $x$ is $p$-nilpotent, there exists an integer $k$ such that

$$
[y, x]=\beta[[y, x], x]=\cdots=\beta^{p^{k}-1}\left[y, x^{p^{k}}\right]=0,
$$

contradicting the fact that $L$ is non-abelian.

Let $m$ be the largest integer such that $x, x^{p}, \ldots, x^{p^{m-1}}$ are linearly independent modulo $\langle y\rangle_{p}$. So there exist coefficients $c_{1}, \ldots, c_{m} \in \mathbb{F}$ such that $\sum_{i=1}^{m} c_{i} x^{p^{i}} \in\langle y\rangle_{p}$. Let $k$ be the smallest integer such that $c_{k} \neq 0$. Then

$$
x^{p^{k}}=-\sum_{i=k+1}^{m}\left(c_{i} / c_{k}\right) x^{p^{i}} \text { modulo }\langle y\rangle_{p} .
$$


It follows from the equation above that $x^{p^{k}} \in\left\langle y, x^{p^{k+1}}\right\rangle_{p}$ and hence $x^{p^{k}} \in\left\langle y, x^{p^{j}}\right\rangle_{p}$, for every $j \geq k$. Since $x$ is $p$-nilpotent, we get $x^{p^{k}} \in\langle y\rangle_{p}$. So, by our choice of $m$, we should have $k=m$. We conclude that there exist another $p$-polynomial $f$ and positive integers $m, n$ such that the following relations hold in $L$ :

$$
\begin{aligned}
& x^{p^{m}}=f(y)=y^{p^{r}}+\cdots, \\
& y^{p^{n}}=0, \\
& {[y, x]=g(y)=\beta_{s} y^{p^{s}}+\cdots, \beta_{s} \neq 0 .}
\end{aligned}
$$

Since $L$ is not abelian, we have $1 \leq r \leq n$ and $1 \leq s \leq n-1$. Now it is easy to see that the commutator subalgebra $L^{\prime}$ is one-dimensional and $\operatorname{cl}(L)=2$. So, $L^{\prime}=\mathbb{F}[x, y] \subseteq L^{p}$. Since $x^{p^{m+n-r}}=y^{p^{n}}=0$, we have $\exp (x)=m+n-r$. Also, $[y, x]^{p^{n-s}}=\beta_{s}^{n-s} y^{p^{n}}=0$. Thus, the exponent of $L^{\prime}$ is equal to $n-s$ which is determined by Lemma 2.5.

Since $L^{\prime} \subseteq L^{p}$, we have $D_{p^{k}}(L)=L^{p^{k}}+L^{\prime p^{k-1}}=L^{p^{k}}$, for every $k \geq 1$. But each quotient $D_{p^{k}}(L) / D_{p^{k+1}}(L)$ is determined by Lemma 2.1. We can then use a similar method as in Lemma 2.5 to show that $\operatorname{dim}_{\mathbb{F}} L^{p^{k}}$ is determined, for every $k \geq 0$. In particular, the exponent of $L$ is determined. So we have:

Lemma 3.1. Let $L \in \mathcal{F}_{p}$ be a non-abelian metacyclic restricted Lie algebra. Then the exponent of $L$ is determined.

We need the following technical lemma in the case $p=2$.

Lemma 3.2. Let $L$ and $H$ be non-abelian restricted Lie algebras over a perfect field of characteristic 2. Suppose that $L$ and $H$ are generated by $x, y$ and $u, v$, respectively, subject to the following relations:

$$
\begin{array}{rlrl}
x^{2}=f\left(y^{2^{r}}\right)=\alpha_{r} y^{2^{r}}+\cdots, & & u^{2}=f\left(v^{2^{r}}\right)+\alpha v^{2^{n-1}}, \\
y^{2^{n}}=0, & v^{2^{n}}=0, \\
{[x, y]=g\left(y^{2^{s}}\right)=\beta_{s} y^{2^{s}}+\cdots,} & \beta_{s} \neq 0, & & {[u, v]=g\left(v^{2^{s}}\right)+\beta v^{2^{n-1}},}
\end{array}
$$

where $f$ and $g$ are some 2 -polynomials, $s \leq r \leq n$, and $\alpha, \beta \in \mathbb{F}$. If $u(L) \cong u(H)$, then $L \cong H$.

Proof. Note that $n \geq 2$, since $L$ is not abelian. Suppose first that $n \geq 3$ and $s<n-1$. We first replace $v$ by $v_{1}=v+\beta^{\prime} v^{2^{n-s-1}}$, where $\beta^{\prime}=\left(\beta / \beta_{s}\right)^{2^{-s}}$. So we get the following relations in $H$ :

$$
u^{2}=f\left(v_{1}^{2^{r}}\right)+\alpha^{\prime} v_{1}^{2^{n-1}}, \quad v_{1}^{2^{n}}=0, \quad\left[u, v_{1}\right]=g\left(v_{1}^{2^{s}}\right) .
$$

We can now replace $u$ by $u_{1}=u+\alpha^{1 / 2} v_{1}^{2^{n-2}}$ and note that the map induced by $x \mapsto u_{1}, y \mapsto v_{1}$ is an isomorphism between $L$ and $H$. Similarly, if $n \geq 3$ and $r=s=n-1$, we can find generators $u_{1}, v_{1}$ of $H$ such that the map induced by $x \mapsto u_{1}, y \mapsto v_{1}$ is an isomorphism. Now we consider the case $n=2$. We have the following relations:

$$
\begin{aligned}
x^{2}=a_{1} y^{2}, & u^{2}=\left(a_{1}+a_{2}\right) v^{2}, \\
y^{4}=0, & v^{4}=0, \\
{[x, y]=b_{1} y^{2}, } & {[u, v]=\left(b_{1}+b_{2}\right) v^{2}, }
\end{aligned}
$$


where $a_{1}, a_{2}, b_{1}, b_{2} \in \mathbb{F}$. Suppose that $\varphi: u(L) \rightarrow u(H)$ is an isomorphism and let $f=\varphi(x)$ and $g=\varphi(y)$. So, $f$ and $g$ generate $u(H), f^{2}=a_{1} g^{2}, g^{4}=0$, and $[f, g]=b_{1} g^{2}$. Let $w=[u, v]$ and take $\{u, v, w\}, u<v<w$, as a basis for $H$. Let $E$ be the vector space spanned by PBW monomials $u v, u w, v w, u v w$. Observe that $E$ is in fact a two-sided ideal of $\omega(H)$ and $E \cap H=0$. Now we express $f$ and $g$ in terms of PBW monomials in $u, v, w$. So we have

$$
f=\alpha_{1} u+\alpha_{2} v+\alpha_{3} w+f_{1}, g=\beta_{1} u+\beta_{2} v+\beta_{3} w+g_{1},
$$

where $f_{1}, g_{1} \in E$. Let $\bar{f}=\alpha_{1} u+\alpha_{2} v+\alpha_{3} w$ and $\bar{g}=\beta_{1} u+\beta_{2} v+\beta_{3} w$. Note that $f^{2}=\bar{f}^{2}+f_{1}^{2}$ modulo $\gamma_{2}\left(\left\langle\bar{f}, f_{1}\right\rangle\right)$. Since $f^{2}=a_{1} g^{2}$ we get

$$
\bar{f}^{2}-a_{1} \bar{g}^{2} \in H \cap E=0 .
$$

Similarly we deduce that $\bar{g}^{4}=0$. Furthermore, $[f, g]=[\bar{f}, \bar{g}]$ modulo $E$ and $g^{2}=\bar{g}^{2}$ modulo $E$. So $[\bar{f}, \bar{g}]-b_{1} \bar{g}^{2} \in H \cap E$ and we get $[\bar{f}, \bar{g}]=b_{1} \bar{g}^{2}$. Since $f, g$ generate $u(H)$, it follows that $\bar{f}, \bar{g}$ generate $u(H) / \omega^{2}(H)$. Hence, $\bar{f}, \bar{g}$ generate $u(H)$ since $\omega(H)$ is nilpotent. Thus, the homomorphism induced by $x \mapsto \bar{f}, y \mapsto \bar{g}$ is an isomorphism between $L$ and $H$.

\section{Proof of the theorem}

Suppose that $L \in \mathcal{F}_{p}$ is a non-abelian metacyclic restricted Lie algebra over a perfect field $\mathbb{F}$. Let $\varphi: u(L) \rightarrow u(H)$ be an algebra isomorphism. Note that $H \in \mathcal{F}_{p}$, since $\omega(L)$ and $\omega(H)$ are nilpotent.

Let $x, y$ be some generators of $L$. By our discussion from Section 3, there exist $p$-polynomials $f, g$ and positive integers $m, n$ such that:

$$
\begin{aligned}
& x^{p^{m}}=f(y)=y^{p^{r}}+\cdots, r \geq 1, \\
& y^{p^{n}}=0, \\
& {[y, x]=g(y)=\beta_{s} y^{p^{s}}+\cdots, s \geq 1 .}
\end{aligned}
$$

We fix the generators $x, y \operatorname{such}$ that $\exp \left(L /\langle y\rangle_{p}\right)=m$ is minimum. Note that if $\exp (x) \leq \exp (y)$, that is, $m \leq r$, we can assume that $s \leq r$. Indeed, if $r<s$, then we replace $x$ by $x^{\prime}=x-y^{p^{r-m}}$ and continue this process if necessary.

Let $t=n-s-1$. Since $c l(L)=2$, we have $\exp \left(L^{\prime}\right)=\exp \left(H^{\prime}\right)=t+1$, by Lemma 2.5. Also, $\operatorname{cl}(H) \leq 3$, by Lemma 2.1. In particular, $H^{\prime}$ is abelian. Hence, by Corollary 2.4, we have

$$
L_{p}^{\prime} / L^{\prime p} \cong H_{p}^{\prime} / H^{\prime p}=\left(H^{\prime}+H^{\prime p}\right) / H^{\prime p} .
$$

Since $L_{p}^{\prime}$ is cyclic, there exists $w \in H^{\prime}$ such that $H_{p}^{\prime}=\mathbb{F} w+H^{\prime p}$. Hence, $H_{p}^{\prime}=\langle w\rangle_{p}$ because $H \in \mathcal{F}_{p}$. Since the ideal $L_{p}^{\prime} u(L)$ is preserved by $\varphi$, we use Lemma 2.6 to see that $\varphi\left(L^{\prime p^{t}} u(L)\right)=H^{\prime p^{t}} u(H)$. So, $\varphi$ induces an isomorphism

$$
u\left(L / L^{\prime p^{t}}\right) \rightarrow u\left(H / H^{\prime p^{t}}\right) .
$$

We may now assume by induction on $\operatorname{dim}_{\mathbb{F}} L$ that $L / L^{\prime p^{t}} \cong H / H^{\prime p^{t}}$. We shall find generators $u, v \in H$ and replace $x$ and $y$ by appropriate generators of $L$, if necessary, so that the map induced by $x \mapsto u, y \mapsto v$ is an isomorphism between $L$ and $H$.

Step 1. $H$ is metacyclic, too. 
Let $u$ and $v$ be some fixed representatives of the images of $x$ and $y$ under the isomorphism $L / L^{\prime p^{t}} \rightarrow H / H^{\prime p^{t}}$. Thus we have the following relations between $u$ and $v$ :

$$
u^{p^{m}}=f(v)+\alpha_{1} w^{p^{t}}, \quad v^{p^{n}}=\alpha_{2} w^{p^{t}}, \quad[v, u]=g(v)+\alpha_{3} w^{p^{t}},
$$

where $H_{p}^{\prime}=\langle w\rangle_{p}$. Note that $c l(H) \leq 3$, by Lemma 2.1. We observe that $u^{p^{m+1}} \in$ $\langle v\rangle_{p}, v^{p^{n+1}}=0$, and $[u, v] \in\langle v\rangle_{p}$. So, $H$ is metacyclic.

Since $\exp \left(H^{\prime}\right)=t+1$, we have $v^{p^{n-1}} \neq 0$. Note that $y^{p^{n-1}}=[y, x]^{p^{t}} \in L^{\prime p^{t}}$. We deduce that $v^{p^{n-1}}=0$ modulo $H^{\prime p^{t}}$. Hence, $v^{p^{n}}=0$. So we can replace (1) by the following relations:

$$
\begin{aligned}
& u^{p^{m}}=f(v)+\alpha v^{p^{n-1}}, \\
& v^{p^{n}}=0, \\
& {[v, u]=g(v)+\beta v^{p^{n-1}} .}
\end{aligned}
$$

Step 2. Assume $\langle x\rangle_{p} \cap\langle y\rangle_{p}=0$.

In other words, $f=0$. So, we have the following relations in $L$ :

$$
\begin{aligned}
& x^{p^{m}}=y^{p^{n}}=0, \\
& {[y, x]=g(y) .}
\end{aligned}
$$

Thus the following relations hold in $H$ :

$$
\begin{aligned}
& u^{p^{m}}=\alpha v^{p^{n-1}}, \\
& v^{p^{n}}=0, \\
& {[v, u]=g(v)+\beta v^{p^{n-1}} .}
\end{aligned}
$$

Suppose $\beta \neq 0$. If $s<n-1$, then we replace $v$ by $v_{1}=v+\beta^{\prime} v^{p^{n-s-1}}$, where $\beta^{\prime}=\left(\beta / \beta_{s}\right)^{p^{-s}}$. Suppose $s=n-1$. Since $L$ and $H$ are non-abelian, we have $\beta+\beta_{s} \neq 0$. Now we replace $u$ by $u_{1}=\beta_{s} u /\left(\beta+\beta_{s}\right)$. So, without loss of generality, we assume that the following relations hold in $H$ :

$$
u^{p^{m}}=\alpha v^{p^{n-1}}, v^{p^{n}}=0,[v, u]=g(v) .
$$

If $m \geq n$, then $\alpha=0$. Otherwise, $\exp (H)=m+1$ whereas $\exp (L)=m$, contradicting Lemma 3.1. If $m \leq n-1$, then we replace $u$ by $u_{1}=u-\alpha^{\prime} v^{p^{n-1-m}}$, where $\alpha^{\prime}=\alpha^{p^{-m}}$. Then we have

$$
u_{1}^{p}=u^{p}-\alpha^{\prime p} v^{p^{n-m}} \text { modulo } \gamma_{p}\left(\left\langle u, v^{p^{n-1-m}}\right\rangle\right) .
$$

So, if $m \geq 2$, then

$$
u_{1}^{p^{m}}=u^{p^{m}}-\alpha v^{p^{n-1}} \text { modulo } \gamma_{p}\left(\left\langle H^{p}, H^{\prime}\right\rangle\right) .
$$

Note that $\gamma_{p}\left(\left\langle H^{p}, H^{\prime}\right\rangle\right)=0$ since $\operatorname{cl}(H)=2$. In the case $m=1$, note that $\gamma_{p}\left(\left\langle u, v^{p^{n-1-m}}\right\rangle\right) \subseteq \gamma_{3}(H)=0$ unless $p=2$ and $m=n-1=1$. So, virtually, $u_{1}^{p^{m}}=0$ and we would be done. The remaining case $p=2$ and $m=n-1=1$ satisfies the hypothesis of Lemma 3.2 and so $L \cong H$.

Step 3. The general case. 
Suppose that $\exp (x) \leq \exp (y)$, that is, $m \leq r$. So, as mentioned earlier, we can assume that $s \leq r$. We replace $x$ by $x_{1}=x-(f(y))^{p^{-m}}$. So, $x_{1}^{p^{m}}=0$ unless $m=r=1$ and $p=2$. We observe that $x_{1}$ and $y$ generate $L$ and $\left\langle x_{1}\right\rangle_{p} \cap\langle y\rangle_{p}=0$. So we would be done by Step 2. In the special case $m=r=1$ and $p=2$, we note that $L \cong H$, by Lemma 3.2. Hence we may assume that $m>r$. Now we have two cases.

Case I. $r \leq s$. Since $x^{p^{m}}=f(y)$, we get $y^{p^{r}} \in\left\langle x^{p^{m}}, y^{p^{r+1}}\right\rangle_{p}$. Thus, $y^{p^{r}} \in$ $\left\langle x^{p^{m}}\right\rangle_{p}$, because $y$ is $p$-nilpotent. Since $r \leq s$, it follows that $[y, x] \in\left\langle x^{p^{m}}\right\rangle_{p}$. Thus, there exists a $p$-polynomial $h$ such that $y^{p^{r}}=h\left(x^{p^{m}}\right)$. Now we replace $y$ by $y_{1}=y-\left(h\left(x^{p^{m}}\right)\right)^{p^{-r}}$. Note that $\exp \left(y_{1}\right)=r<m$. So we have found new generators $x^{\prime}=y_{1}, y^{\prime}=x$ such that $\exp \left(L /\left\langle y^{\prime}\right\rangle_{p}\right)<m$. This contradicts the minimality of $m$. So Case I is not possible and we have to consider the case $r>s$.

Case II. $r>s$.

If $r=n-1$, then $x^{p^{m}}=y^{p^{n-1}}$. We replace $y$ by $y_{1}=y-x^{p^{m-n+1}}$. Observe that $\langle x\rangle_{p} \cap\left\langle y_{1}\right\rangle_{p}=0$ and so we are done by Step 2. It remains to consider the case $r<n-1$. In this case we replace $x$ by $x_{1}=x+\alpha^{\prime} x^{p^{n-r-1}}$, where $\alpha^{\prime}=\alpha^{p^{-m}}$. We get the following relations in $L$ :

$$
x_{1}^{p^{m}}=f(y)+\alpha y^{p^{n-1}}, \quad y^{p^{n}}=0, \quad\left[y, x_{1}\right]=g(y) .
$$

Note that $s<n-1$. Now we replace $y$ by $y_{1}=y-\beta^{\prime} y^{p^{n-s-1}}$, where $\beta^{\prime}=\left(\beta / \beta_{s}\right)^{p^{-s}}$. Note that $\left[x_{1}, y\right]=\left[x_{1}, y_{1}\right]$. Also, we observe that $g\left(y_{1}\right)=g(y)-\beta y^{p^{n-1}}$. Thus,

$$
\left[y_{1}, x_{1}\right]=\left[y, x_{1}\right]=g(y)=g\left(y_{1}\right)+\beta y_{1}^{p^{n-1}} .
$$

Furthermore, $x_{1}^{p^{m}}=f\left(y_{1}\right)+\alpha y_{1}^{p^{n-1}}$, since $r>s$. So, we get the following relations in $L$ :

$$
\begin{aligned}
& x_{1}^{p^{m}}=f\left(y_{1}\right)+\alpha y_{1}^{p^{n-1}}, \\
& y_{1}^{p^{n}}=0, \\
& {\left[y_{1}, x_{1}\right]=g\left(y_{1}\right)+\beta y_{1}^{p^{n-1}} .}
\end{aligned}
$$

We deduce that the map induced by $x_{1} \mapsto u, y_{1} \mapsto v$ is an isomorphism between $L$ and $H$.

\section{REFERENCES}

[1] C. Bagiński, A. Konovalov, The modular isomorphism problem for finite $p$-groups with a cyclic subgroup of index $p^{2}$, Groups St. Andrews 2005. Vol. 1, 186-193, London Math. Soc. Lecture Note Ser., 339 (Cambridge Univ. Press, 2007). MR2327323 (2008f:20009)

[2] C. Bagiński, The isomorphism question for modular group algebras of metacyclic $p$-groups, Proc. Amer. Math. Soc. 104 (1988), no. 1, 39-42. MR958039 (89i:20016)

[3] N. Jacobson, Lie Algebras (Interscience, New York, 1962). MR0143793 (26:1345)

[4] D.M. Riley, A. Shalev, Restricted Lie algebras and their envelopes, Canad. J. Math., 47 (1995), 146-164. MR.1319694 (96e:16031)

[5] D.M. Riley, A. Shalev, The Lie structure of enveloping algebras, J. Algebra, 162 (1993), no. 1, 46-61. MR.1250526 (94k:17019)

[6] D.M. Riley, H. Usefi, The isomorphism problem for universal enveloping algebras of Lie algebras, Algebras and Representation Theory, 10 (2007), no. 6, 517-532. MR.2350224

[7] R. Sandling, The modular group algebra problem for metacyclic $p$-groups, Proc. Amer. Math. Soc., 124 (1996), no. 5, 1347-1350. MR.1343723 (96g:20003) 
[8] R. Sandling, The isomorphism problem for group rings: a survey, Orders and Their Applications (Oberwolfach, 1984), Lecture Notes in Math., 1142 (Springer, Berlin, 1985), 256-288. MR 812504 (87b:20007)

[9] H. Strade, R. Farnsteiner, Modular Lie Algebras and Their Representations, Monographs and Textbooks in Pure and Applied Mathematics, 116 (Marcel Dekker, New York, 1988). MR.929682(89h:17021)

[10] H. Usefi, Isomorphism invariants of restricted enveloping algebras, preprint is available on arXiv:0804.2281.

Department of Mathematics, University of British Columbia, 1984 Mathematics Road, Vancouver, BC, Canada, V6T $1 \mathrm{Z} 2$

E-mail address: usefi@math.ubc.ca 